\title{
Immediate versus conventional loading (IL vs. CL) influence on implant marginal bone loss (MBL): an overview
}

\author{
Rebeca Serrano Zamora ${ }^{1}$, Óscar Iglesias Velázquez ${ }^{1}$, Baoluo Xing Gao ${ }^{1}$, \\ Carmen Martín Carreras-Presas ${ }^{2} \wedge$, Juan López-Quiles ${ }^{1} \wedge$ \\ ${ }^{1}$ Department of Dental Clinical Specialties, Faculty of Odontology, Complutense University, Madrid, Spain; ${ }^{2}$ Faculty of Odontology, European \\ University, Madrid, Spain \\ Contributions: (I) Conception and design: R Serrano Zamora, Ó Iglesias Velázquez; (II) Administrative support: BX Gao; (III) Provision of study materials \\ or patients: J López-Quiles, C Martín Carreras-Presas; (IV) Collection and assembly of data: R Serrano Zamora, Ó Iglesias Velázquez; (V) Data analysis \\ and interpretation: R Serrano Zamora, Ó Iglesias Velázquez; (VI) Manuscript writing: All authors; (VII) Final approval of manuscript: All authors. \\ Correspondence to: Rebeca Serrano Zamora. Department of Dental Clinical Specialties, Faculty of Dentistry, Complutense University, Plaza Ramón y \\ Cajal, s/n (Ciudad Universitaria), 28040 Madrid, Spain. Email: rebese01@ucm.es.
}

\begin{abstract}
This article aims to determine whether the implant loading time influences the marginal bone loss (MBL) that occurs in the different restorations on dental implants. Different concepts of prosthetic loading have been proposed in literature. While the conventional loading (CL) protocol dictates to wait for a period of osseointegration of 3-4 to 6-8 months before loading the implant, the immediate loading (IL) protocol proposes to perform the implant loading within a period of no more than 1 week after its placement. Although there are several parameters to evaluate implant success, the evaluation of the radiographic changes of the MBL with standardized intraoral radiographic is the most used method in the literature to evaluate the success of the implant over time. A comprehensive search of the literature was conducted in the PubMed/ MEDLINE (National Library of Medicine), Cochrane Library, Scopus and Web of Science electronic databases. The search strategy was done using the following terms: "dental implants", "loading protocols", "MBL". MBL is similar in both protocols, with no significant differences found in most published studies. IL has certain advantages such as reduced treatment time. However, good primary stability is needed to perform it. More studies are needed to establish clear conclusions.
\end{abstract}

Keywords: Dental implants; loading protocols; marginal bone loss (MBL)

Received: 21 April 2021; Accepted: 22 July 2021; Published: 30 December 2022.

doi: $10.21037 /$ fomm-21-51

View this article at: https://dx.doi.org/10.21037/fomm-21-51

\section{Introduction}

Since dental implants were created, different concepts of prosthetic loading have been proposed in literature (1). The International Team for Implantology, have recently recommended three different loading protocols according to the loading period, defined as conventional, early and immediate (2).

The conventional protocol proposed by Brånemark in 1977 consisted on waiting for a healing period of at least 6 months before implant placement with the aim of performing implant surgery on healed sites (3). After that, the conventional loading (CL) protocol dictates to wait before loading the implant for a period of osseointegration ranging from 3-4 to 6-8 months for implants placed in the mandible or in the maxillary bone respectively. The early loading (EL) protocol is based on loading the implant between 1 week and 2 months after implant

^ ORCID: Rebeca Serrano Zamora, 0000-0002-1936-7115; Óscar Iglesias Velázquez, 0000-0002-1330-4885; Carmen Martín CarrerasPresas, 0000-0002-0937-0994; Juan López-Quiles, 0000-0002-5817-0526. 
placement and the immediate loading (IL) protocol loads the implant within a period of no more than 1 week after its placement (2).

According to Brånemark, premature loading could affect the osseointegration process by creating fibrous tissue between the bone and the implant surfaces, altering the healing period and causing premature mobility and implant failure $(2,4,5)$. Recent developments have modified the dimensions, materials, macro and microstructure of the implant, improving the achievement of primary stability, shortening healing times and enhancing osseointegration $(6,7)$. However, due to the lack of long-term data supporting these protocols, it is still premature to consider them clinically acceptable (8).

There are several parameters to evaluate implant success, of which peri-implant bone and soft tissue stability are some of the most important factors (3). Marginal bone loss (MBL) has been used in literature as an important tool for the evaluation of success rates on implants, accepting a maximum bone resorption of $1.5 \mathrm{~mm}$ during the first year and $0.2 \mathrm{~mm}$ annually. Higher resorption rates will eventually lead to implant failure, because it interferes with the stability of surrounding tissues and causes peri implantitis or mobility of the implant $(1,4)$. The evaluation of the radiographic changes of the bone level with standardized intraoral radiographic methods is the most used method in the literature to evaluate the success of the implant over time (8).

The objective of our review is to determine whether the implant loading time influences the MBL that occurs in the different restorations on dental implants.

\section{Methods}

A comprehensive search of the literature was conducted in the PubMed/MEDLINE (National Library of Medicine), Cochrane Library, Scopus and Web of Science electronic databases. The search strategy was done using the following terms: "dental implants", "loading protocols", "MBL".

Only articles in English were considered. No time restrictions were applied. Literature and systematic reviews, clinical trials and observational studies were contemplated. Studies with full-text were selected and reviewed.

\section{Discussion}

As a result of the evolution of Oral Implantology, dental implants achieved better osseointegration by modifying their properties. These advances have resulted in higher primary stability and enhanced implant prognosis. Consequently, loading protocols have been modified from CL to earlier and even IL $(6,7)$.

Primary stability is the most important consideration in applying immediate implant loading protocol. IL protocol began to be applied to reduce the waiting time between implant placement surgery and prosthetic rehabilitation and to improve patient's quality of life. IL protocol has many advantages such as quicker achievement of occlusal function, early restoration of esthetic appearance and absence of a second surgery. However, an increased risk of infection and insufficient volume are challenges that clinicians may deal with this protocol (9). Nowadays, IL is one of the most commonly used protocols, especially in anterior maxilla and mandibles with good bone quality $(5,7)$. Despite the increase in its implementation in the last years, there is still some controversy due to the uncertain outcomes when compared to conventional protocol (2).

One of the parameters used to evaluate the success of implants treatment is MBL. The etiology of bone loss is multifactorial, including biomechanical and biological factors (10). All factors influencing marginal bone levels have not yet been determined. However, it has been proposed that the loading protocol may influence the MBL observed (8). Excessive peri-implant bone loss can compromise esthetics outcomes and especially cause implant failure $(11,12)$.

Nowadays, X-ray evaluation is the only method to evaluate bone level changes around dental implants. Therefore, in order to compare studies and establish correct conclusions, it is necessary to use standardized radiographic methods. While periapical radiographs and Cone beam computed tomography (CBCTs) are acceptable for measurement, orthopantomography distorts the images in the anterior region (8).

To evaluate the MBL resulting from immediate or CL, the type of prosthetic restoration performed must be considered.

\section{MBL in fixed prosthesis}

Numerous studies and systematic reviews published in the last years have analyzed MBL around implants rehabilitated with fixed prostheses (single crowns, partial restorations, full-arch restorations...). Fixed prostheses often last longer and have fewer complications than overdentures (10).

In a recent systematic review published by Cheng et al., 
a total of 386 single implants (189 immediately loaded and 197 conventionally loaded) were included. The mean difference in MBL was $0.03 \mathrm{~mm}$ at 1 year and $-0.01 \mathrm{~mm}$ 2 years of follow-up. No significant differences were found between both loading implant protocols (7). Similar results were obtained in the meta-analysis performed by Chen et al. A total of 3,746 implants were placed. MBL ranged from -1.32 to $0 \mathrm{~mm}$ in the IL protocol group and from -1.25 to $-0.10 \mathrm{~mm}$ in the CL protocol group. The result showed no statistically significant difference in crestal bone loss $(\mathrm{P}=0.645)$ (13). The studies reviewed by Moraschini et al. also found no significant differences in MBL when comparing IL (0.4 to $1.31 \mathrm{~mm}) v s$. CL protocols (0.68 to $1.2 \mathrm{~mm})(\mathrm{P}=0.24)(14)$. In the same way, Benic et al. also did not find significant differences in MBL in 7 of the 10 randomized clinical trials (RCTs) included in their review comparing both loading protocols after one year of follow up. Again, no statistical differences were found after 2, 3 and 5 years of loading (3).

The meta-analysis reported by Suarez et al. included 321 immediately loaded implants and 192 conventionally loaded implants. The mean difference in MBL between IL and CL was $-0.09 \mathrm{~mm}$, favorable to the IL group without statistically significant $(\mathrm{P}=0.33)$. The funnel plot showed asymmetric distributions, indicating the possibility of publication bias. Because most articles did not present significant differences, the funnel plot results are not representative (15). In a similar way, the meta-analysis performed by Sanz-Sánchez et al., obtained a higher MBL with statistically significant differences in the CL protocol when compared with the IL protocol in those studies that included fixed implant-supported restorations (11).

For some authors, despite the high survival rate (95$100 \%$ ) of the implants, the heterogeneity of the studies does not allow to establish a strong conclusion (2).

\section{MBL in removable prostheses (overdentures)}

Implant retained overdentures are removable prosthesis that are widely used, especially in edentulous patients with severe alveolar resorption, loss of soft tissues support and difficulty in maintaining adequate oral hygiene (16).

Although CL protocol has been performed in most of the studies, IL has also begun to be performed on implantretained overdentures. This protocol has been shown to be a successful treatment option. However, the benefits associated with each loading protocol are still unclear, especially in older edentulous patients, who are the main carriers of implant-retained overdentures. Several factors may compromise the osseointegration process in these patients, such as systemic diseases, bone quality, degree of atrophy, etc.

Schuster et al. reported a recent RCT in which 20 patients received two mandibular implants to be rehabilitated with implant-retained overdentures. The loading protocol (CL or IL) was randomized and MBL was analyzed. No statistically significant differences were found in MBL between groups after 1-year follow-up ( $>0.05)$. The mean MBL for the conventional and IL group was similar, about $-0.05 \mathrm{~mm}$ (17). The results of this study agreed with some systematic reviews. In the meta-analysis performed by Sanz-Sánchez et al., no significant differences were found between IL and CL in implants rehabilitated with overdentures (11). In a recent systematic review published by Borges et al., a total of 841 participants and 1,874 implants were included. The study group was formed by implants loaded immediately or early (1,046 implants), and the control group by those placed conventionally (780 implants). Different overdenture designs and attachments were included and evaluated. The MBL means in implants installed with IL protocol or EL protocol were similar to those implants installed with CL protocol at $6,12,24$ and 36 months of follow up. In this systematic review, no significant differences were found in the analysis of MBL in any of the loading protocols, demonstrating similar success and survival rates (6). These results are also in concordance with those obtained in the systematic review reported by Sanda et al., who concluded that there were no significant differences in MBL around implants supporting overdentures, independently of the loading protocol (18).

However, different results have been published in the literature. Ma et al. studied a cohort of 106 patients with mandibular implant-retained overdentures. The participants were randomly divided into three groups according to the loading protocol (IL, EL, CL). In contrast to the previous mentioned studies, there was a higher MBL with the IL protocol, which was statistically significant compared with the EL and CL protocols during the first year. The IL group $(0.48 \pm 0.62 \mathrm{~mm})$ also showed a higher MBL at 10 years follow-up of the study (8). In the RCT performed by Elsyad et al., the IL group was also associated with more marginal bone resorption when compared with CL of implants after 3 years (19).

Yet, in other studies, mesial and distal MBL increased in cases where implant loading was delayed (1). In the systematic review performed by Sommer et al., a total of 
2,336 implants supporting fixed prostheses and removable prostheses were reviewed and differences in MBL were found when comparing loading protocols. The results of the meta-analysis showed a mean MBL of $0.457 \mathrm{~mm}$ for IL, $0.390 \mathrm{~mm}$ for immediate non occlusal loading, $0.488 \mathrm{~mm}$ for EL ( $>2$ days to $<3$ months) and $0.852 \mathrm{~mm}$ for CL. CL showed a significantly higher MBL than the other loading protocols (12).

In the research conducted by Kutkut et al., MBL was also compared between IL and CL for mandibular implant-retained overdentures. Twenty patients were randomly divided into IL or CL protocol. There were significant changes in bone level between baseline and 12 months for the IL $(0.65 \pm 1.69)$ and CL groups $(-1.33 \pm 1.473)(\mathrm{P}=0.009)(20)$.

In the meta-analysis conducted by Esposito et al. in 2013, a total of nine articles were analyzed, comparing MBL in immediately and conventionally loaded implants. Rehabilitations in fixed and removable prostheses were included. The result of the meta-analysis determined reduced bone loss associated with immediately loaded implants $(-0.10 \mathrm{~mm})(\mathrm{P}=0.03)$. Nevertheless, authors consider that quality of the evidence as very low due to high and unclear risk of bias of selected studies and reports that the small difference in MBL between protocols may not be clinically important (5).

According to the results of the literature review, the success and survival rates of dental implants loaded immediately or conventionally are similar. Overall, there was no significant evidence of a clinically important difference concerning implant failure and MBL associated with different implant loading protocols.

The application of the IL protocol is a reasonable alternative to the CL protocol. In addition, it would reduce treatment time, which could increase patient satisfaction. Still, the use of the IL protocol is still not explored enough in patients with implant-retained overdentures. Moreover, the results of the literature in relation to implant-retained removable prostheses are more heterogeneous. Therefore, more comparisons between IL and CL are needed.

Other factors that could affect MBL should also be considered in order to compare future studies outcomes. For instance, authors discuss if immediately loaded implants should have occlusion. A recent meta-analysis suggests that immediately occlusally loaded implants and immediately non-occlusally loaded implants are not associated with clinical outcomes in terms of MBL. Non-occlusally loaded implants are considered those implants that are immediately rehabilitated with provisional restorations without occlusal contacts with the opposing dentition, preventing centric or lateral excursions to ensure an appropriate healing although pressure from soft tissues cannot be avoided. The absence of excessive implant micromobility is considered absolutely necessary for osseointegration. A micromovement of more than $100 \mu \mathrm{m}$ is sufficient to compromise osseointegration with fibrous encapsulation formation $(5,7)$.

There are several types of dental implants, with different macrostructure and microstructure and different designs. In some systematic reviews, such as that of Esposito et al, the type of implant is analyzed. More studies are needed to determine if the implant design is responsible for the MBL (5).

Another factor that should be considered and differs among the studies is the surgical technique. No significant differences were found between IL and CL in flap surgery although differences were found between both protocols in flapless surgeries (13).

In relation to overdenture rehabilitations, the type of attachment selected should be considered (Locator, Equator, magnetic, bar and ball). Some studies have compared IL and CL protocols and the association between MBL and with the type of attachment selected. In the study of Ma et al., there were no statistically significant differences between the different overdenture attachments at year 5 (8).

Only studies published in English were included in this review, which could be considered a possible limitation for this study. Moreover, more RCTs should be performed including more sample size and long term follow up. Furthermore, future studies should use standardized methodology (surgical technique, measurement of bone loss, etc.) to determine the MBL comparing IL and CL protocols.

\section{Summary}

The International Team for Implantology, have recently recommended three different loading protocols defined as conventional, early and immediate. The CL protocol dictates to wait before loading the implant for a period of osseointegration ranging from 3-4 to 6-8 months for implants placed in the mandible or in the maxillary bone respectively. In the immediate protocol, the implants must be loaded in less than one week after surgery.

MBL has been used in literature as an important tool for the evaluation of success rates on implants, accepting a maximum bone resorption of $1.5 \mathrm{~mm}$ during the first year 
and $0.2 \mathrm{~mm}$ annually.

According to the results of the literature review, the success and survival rates of dental implants loaded immediately or conventionally are similar. The application of the IL protocol is a reasonable alternative to the CL protocol. Overall, there is not significant evidence of a clinically important difference concerning implant failure and MBL associated with different implant loading protocols.

More RCTs should be performed including more sample size and long-term follow-up. Furthermore, future studies should use standardized methodology to determine the MBL comparing IL and CL protocols.

\section{Acknowledgments}

Funding: None.

\section{Footnote}

Provenance and Peer Review: This article was commissioned by the Guest Editor (Mario Pérez-Sayáns) for the series "Marginal Bone Loss in Dental Implants: Factors Affecting and How to Prevent It" published in Frontiers of Oral and Maxillofacial Medicine. The article has undergone external peer review.

Conflicts of Interest: All authors have completed the ICMJE uniform disclosure form (available at https://fomm. amegroups.com/article/view/10.21037/fomm-21-51/coif). The series "Marginal Bone Loss in Dental Implants: Factors Affecting and How to Prevent It" was commissioned by the editorial office without any funding or sponsorship. The authors have no other conflicts of interest to declare.

Ethical Statement: The authors are accountable for all aspects of the work in ensuring that questions related to the accuracy or integrity of any part of the work are appropriately investigated and resolved.

Open Access Statement: This is an Open Access article distributed in accordance with the Creative Commons Attribution-NonCommercial-NoDerivs 4.0 International License (CC BY-NC-ND 4.0), which permits the noncommercial replication and distribution of the article with the strict proviso that no changes or edits are made and the original work is properly cited (including links to both the formal publication through the relevant
DOI and the license). See: https://creativecommons.org/ licenses/by-nc-nd/4.0/.

\section{References}

1. Bilhan H, Mumcu E, Arat S. The role of timing of loading on later marginal bone loss around dental implants: a retrospective clinical study. J Oral Implantol 2010;36:363-76.

2. Gallardo YNR, da Silva-Olivio IR, Gonzaga L, et al. A Systematic Review of Clinical Outcomes on Patients Rehabilitated with Complete-Arch Fixed ImplantSupported Prostheses According to the Time of Loading. J Prosthodont 2019;28:958-68.

3. Benic GI, Mir-Mari J, Hämmerle CH. Loading protocols for single-implant crowns: a systematic review and meta-analysis. Int J Oral Maxillofac Implants 2014;29 Suppl:222-38.

4. Kim YK, Ahn KJ, Yun PY, et al. Effect of loading time on marginal bone loss around hydroxyapatite-coated implants. J Korean Assoc Oral Maxillofac Surg 2013;39:161-7.

5. Esposito M, Grusovin MG, Maghaireh H, et al. Interventions for replacing missing teeth: different times for loading dental implants. Cochrane Database Syst Rev 2013;(3):CD003878.

6. Borges GA, Costa RC, Nagay BE, et al. Long-term outcomes of different loading protocols for implantsupported mandibular overdentures: A systematic review and meta-analysis. J Prosthet Dent 2021;125:732-45.

7. Cheng Q, Su YY, Wang X, et al. Clinical Outcomes Following Immediate Loading of Single-Tooth Implants in the Esthetic Zone: A Systematic Review and MetaAnalysis. Int J Oral Maxillofac Implants 2020;35:167-77.

8. Ma S, Tawse-Smith A, Thomson WM, et al. Marginal bone loss with mandibular two-implant overdentures using different loading protocols and attachment systems: 10year outcomes. Int J Prosthodont 2010;23:321-32.

9. Bassir SH, El Kholy K, Chen CY, et al. Outcome of early dental implant placement versus other dental implant placement protocols: A systematic review and metaanalysis. J Periodontol 2019;90:493-506.

10. Misch CE. Dental implant prosthetics. 2nd edition. St. Louis: Elsevier Mosby, 2015.

11. Sanz-Sánchez I, Sanz-Martín I, Figuero E, et al. Clinical efficacy of immediate implant loading protocols compared to conventional loading depending on the type of the restoration: a systematic review. Clin Oral Implants Res 2015;26:964-82. 
12. Sommer M, Zimmermann J, Grize L, et al. Marginal bone loss one year after implantation: a systematic review of different loading protocols. Int J Oral Maxillofac Surg 2020;49:121-34.

13. Chen J, Cai M, Yang J, et al. Immediate versus early or conventional loading dental implants with fixed prostheses: A systematic review and meta-analysis of randomized controlled clinical trials. J Prosthet Dent 2019;122:516-36.

14. Moraschini V, Porto Barboza E. Immediate versus conventional loaded single implants in the posterior mandible: a meta-analysis of randomized controlled trials. Int J Oral Maxillofac Surg 2016;45:85-92.

15. Suarez F, Chan HL, Monje A, et al. Effect of the timing of restoration on implant marginal bone loss: a systematic review. J Periodontol 2013;84:159-69.

16. Laverty DP, Green D, Marrison D, et al. Implant retention systems for implant-retained overdentures. Br Dent J 2017;222:347-59.

17. Schuster AJ, Marcello-Machado RM, Bielemann AM, et

doi: $10.21037 /$ fomm-21-51

Cite this article as: Serrano Zamora R, Iglesias Velázquez Ó, Gao BX, Martín Carreras-Presas C, López-Quiles J. Immediate versus conventional loading (IL vs. CL) influence on implant marginal bone loss (MBL): an overview. Front Oral Maxillofac Med 2022;4:36. al. Immediate vs conventional loading of Facility-Equator system in mandibular overdenture wearers: 1-year RCT with clinical, biological, and functional evaluation. Clin Implant Dent Relat Res 2020;22:270-80.

18. Sanda M, Fueki K, Bari PR, et al. Comparison of immediate and conventional loading protocols with respect to marginal bone loss around implants supporting mandibular overdentures: A systematic review and metaanalysis. Jpn Dent Sci Rev 2019;55:20-5.

19. Elsyad MA, Al-Mahdy YF, Fouad MM. Marginal bone loss adjacent to conventional and immediate loaded two implants supporting a ball-retained mandibular overdenture: a 3-year randomized clinical trial. Clin Oral Implants Res 2012;23:496-503.

20. Kutkut A, Rezk M, Zephyr D, et al. Immediate Loading of Unsplinted Implant Retained Mandibular Overdenture: A Randomized Controlled Clinical Study. J Oral Implantol 2019;45:378-89. 